\title{
Microscopic Basis for the Mechanism of Carrier Dynamics in an Operating $p$ - $n$ Junction Examined by Using Light-Modulated Scanning Tunneling Spectroscopy
}

\author{
Shoji Yoshida, Yuya Kanitani, Ryuji Oshima, Yoshitaka Okada, Osamu Takeuchi, and Hidemi Shigekawa* \\ Institute of Applied Physics, CREST-JST, 21st COE, University of Tsukuba, Tsukuba 305-8573 Japan
}

(Received 7 July 2006; published 10 January 2007)

\begin{abstract}
The doping characteristics and carrier transport in a GaAs $p$ - $n$ junction were visualized with a $\sim 10 \mathrm{~nm}$ spatial resolution, using light-modulated scanning tunneling spectroscopy. The dynamics of minority carriers under operating conditions, such as recombination, diffusion, and electric field induced drift, which had previously been analyzed on the basis of empirical electric properties, were successfully examined on the nanoscale. These results provide a solid basis for elucidating the mechanism of the carrier transport properties predicted by using the macroscopic analysis.
\end{abstract}

DOI: $10.1103 /$ PhysRevLett.98.026802

PACS numbers: 87.64.Dz, 73.40.Kp, 73.43.Fj

The dynamics of the doped minor carriers plays an essential role in the functional materials and devices. With the miniaturization of semiconductor devices, for example, the difference in such electronic properties of each element has an evermore crucial influence on macroscopic functions. However, the electronic properties have been, in general, analyzed using the macroscopic behavior obtained by the data averaged over the operating area. Thus, direct observation of the characteristics, which provides us with the basis of the results of the macroscopic analysis, is of great importance and there has been a growing desire to obtain nanoscale characterization techniques to probe various electrical properties in semiconductor devices. For this purpose, advanced microscopy, which enables us to investigate nanoscale electrostatic potential distributions and doping profiles, has been developed [1-4]. However, the measurement of carrier transport in operating devices has rarely been performed $[5,6]$ in spite of its fundamental and practical importance.

In this Letter, we present the results of real-space mapping of the doping characteristics and carrier transport in an operating semiconductor device, a GaAs $p$ - $n$ junction, using light-modulated scanning tunneling spectroscopy (LM-STS) [7]. The characteristics of the minority-carrier flow into the neutral region were successfully visualized, providing the solid basis for elucidating the mechanism of the carrier transport properties predicted by using the macroscopic analysis.

LM-STS was employed to measure the surface photovoltage (SPV) of a semiconductor material below the scanning tunneling microscopy (STM) tip. SPV is defined as the change in the surface potential of the semiconductor material induced by super-band-gap illumination [Fig. 1(c)], which provides information about the polarity and magnitude of the surface band bending under the dark condition $[8,9]$. In STM measurement, since the bias voltage applied between the tip and the sample modifies the amount of surface band bending, the obtained SPV includes bias-dependent characteristics [10]. This phenomenon is called tip-induced band bending (TIBB), the value of which is governed by the doping characteristics [11] and the dynamical behavior of the excess carriers injected, for example, by the external bias voltage and optical excitations. By taking advantage of these characteristics, we quantitatively determined the doping profile across a $p-n$ junction interface with a $\sim 10 \mathrm{~nm}$ resolution, and succeeded in examining the minority-carrier dynamics on the nanoscale under an operating condition.

The experimental setup is illustrated in Fig. 1(a). A $p-n$ junction was prepared by growing $n$-(Si-doped, $2.0 \times$ $10^{18} \mathrm{~cm}^{-3}, 500 \mathrm{~nm}$ ) and $p$-type (Be-doped, $2.0 \times$ $\left.10^{18} \mathrm{~cm}^{-3}, 500 \mathrm{~nm}\right) \mathrm{GaAs}$ layers on an $n^{+}-\mathrm{GaAs}(001)$

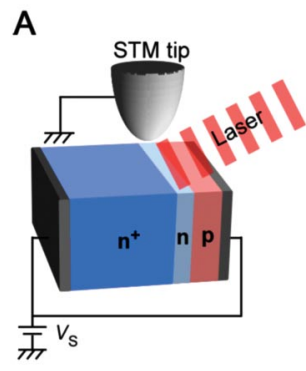

C

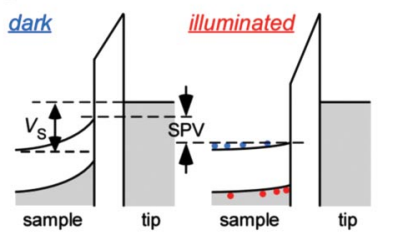

$B$

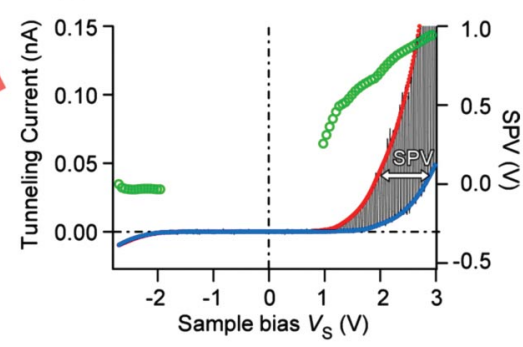

D

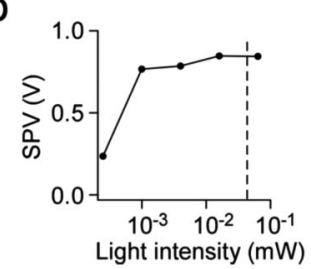

FIG. 1 (color). (a) Experimental setup. (b) $I-V$ curve (left axis) and SPV spectrum (right axis) obtained on the $n$-type area (set point: $V_{S}=+3.0 \mathrm{~V}, I=0.10 \mathrm{nA}$ ). (c) $1 \mathrm{D}$ energy diagrams of the tunnel junction for an $n$-type semiconductor with a positive sample bias voltage under dark (left) and illuminated (right) conditions. When electron (blue circles)-hole (red circles) pairs are produced by super-band-gap illumination, holes flow towards the surface due to the electric field affecting the area, resulting in the reduction of band bending. (d) Light-intensity dependence of SPV measured on an $n$-type area $\left(V_{S}=+2.5 \mathrm{~V}\right)$. 
substrate (Si-doped, $8.3 \times 10^{18} \mathrm{~cm}^{-3}$ ) using molecular beam epitaxy [12]. Au-Zn Ohmic-contact electrodes were formed on both sides of the samples. LM-STS measurements were performed on a cleaved clean (110) surface at room temperature in ultrahigh-vacuum $\left(<1 \times 10^{-8} \mathrm{~Pa}\right)$. Mechanically chopped illumination from a laser diode $(635 \mathrm{~nm}, 100 \mathrm{~Hz}), 60^{\circ}$ off-normal to the surface, was focused onto the tunnel junction area with a spot diameter of $0.02 \mathrm{~mm}$. A tungsten STM tip was used for measurement.

Figure 1(b) shows a typical current-voltage spectrum ( $I-V$ curve) acquired on the $n$-type area. Here, both the electrodes were shorted, and sample bias voltage $V_{S}$ was applied via the two electrodes, as shown in Fig. 1(a) (STM tip is grounded). For the positive-sample-bias-voltage region, the tunneling current oscillates due to the chopped laser illumination, and the two virtual $I-V$ curves, which correspond to those under dark (blue) and illuminated (red) conditions, can be simultaneously obtained. The SPV spectrum (green circles) is obtained by calculating the lateral shift of the two $I-V$ curves with respect to the bias voltage for the $I-V$ curve under the dark condition. The central part of the spectrum with the tunneling current below $3 \mathrm{pA}$ is missing due to the difficulty in calculating the shift of the two $I-V$ curves in that region.

Since the GaAs(110) surface has no surface state within the bulk band gap, TIBB easily occurs for the positive $V_{S}$. In contrast, for the negative sample bias voltage condition, the SPV is small because the Fermi level is close to the valence band edge, as shown in the onedimensional (1D) energy diagram of the tunneling junction, which has a metal-insulator-semiconductor (MIS)

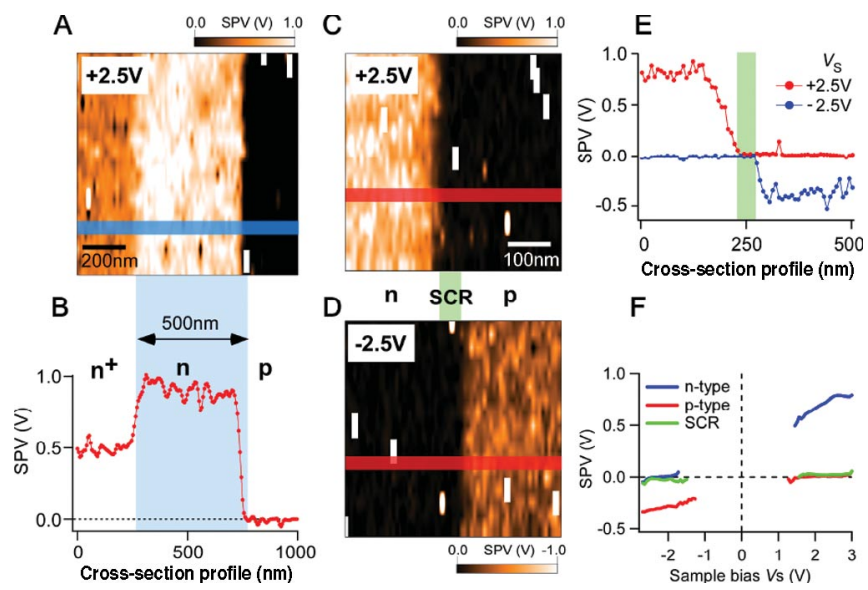

FIG. 2 (color). (a) SPV mapping at $V_{S}=+2.5 \mathrm{~V}(1000 \mathrm{~nm} \times$ $1000 \mathrm{~nm}, 80$ (lateral) $\times 20$ (vertical) LM-STS spectra). (b) A plot of the cross-section along the blue line marked in (a). (c), (d) are SPV mappings of the same area at $V_{S}=+2.5 \mathrm{~V}$ and $V_{S}=$ $-2.5 \mathrm{~V}$, respectively $(500 \mathrm{~nm} \times 500 \mathrm{~nm}, 67 \times 17$ LM-STS spectra). (e) Cross sections of the SPV images along the red lines in (c) - red dots, (d) - blue dots. Green bar represents the space charge region (SCR) at the interface. (f) SPV spectra obtained by averaging all data in each region (blue: $n$-type region, green: space charge region, red: $p$-type region). structure [Fig. 1(c)]. For the measurement on the $p$-type area, the opposite bias dependence occurs.

In order to obtain the flat band condition during photoillumination, we illuminated the sample with light of the intensity indicated by the dashed line in Fig. 1(d). By making the light spot small, the thermal expansion [9] effect was reduced, which was ascertained by confirming that there was no photoresponse in the tunneling current on the $n$-type area under the negative $V_{S}$, where no SPV is expected as mentioned earlier. Under these conditions, the measured SPV is equivalent to the amount of TIBB existing under the dark condition.

Spatially resolved LM-STS measurements were performed simultaneously with topographic imaging. The feedback loop of STM was opened at equally spaced measurement points during topographic imaging to fix the tip position and an $I-V$ curve was acquired under chopped light at each point. As explained using Fig. 1(b), the SPV was calculated from the $I-V$ curves for the chosen bias voltages and shown with a color scale in Figs. 2(a), 2(c), 2(d), and 3(b).

Before demonstrating the visualized carrier dynamics under operating conditions, we first show the potential of this method by presenting the results obtained under the
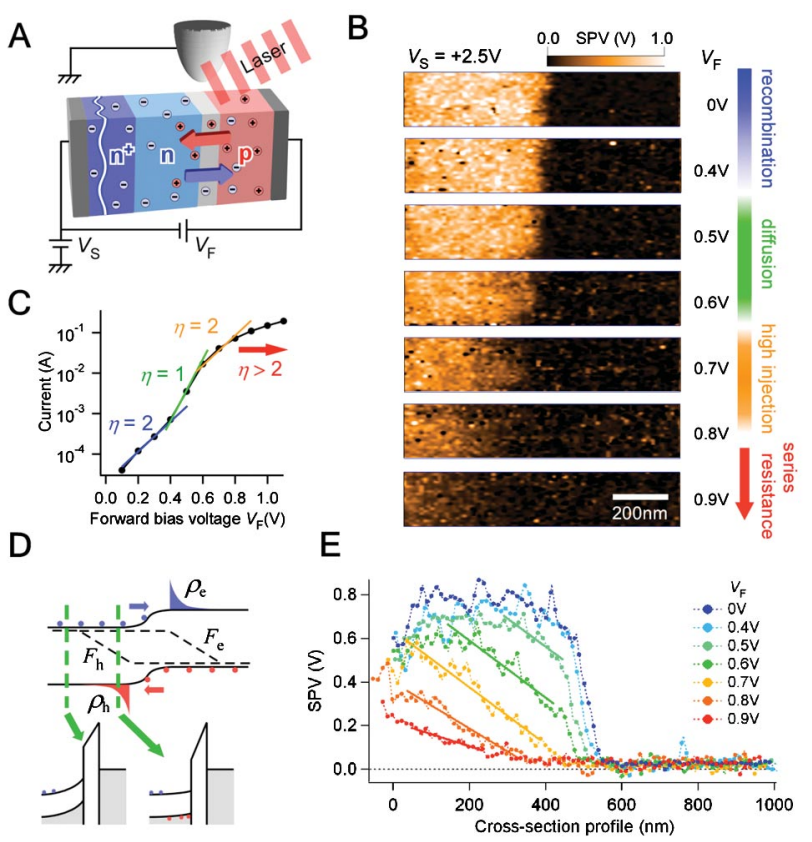

FIG. 3 (color). (a) Experimental setup. (b) A series of SPV mappings as a function of the applied forward-bias voltage $\left(K_{S}=+2.5 \mathrm{~V}\right)$. (c) A current-voltage relation macroscopically obtained for the $p-n$ junction. (d) (upper) Band diagram of a $p-n$ junction with the distribution of minority electrons (blue circles) and holes (red circles) under a forward-biased voltage condition. $F_{h}$ and $F_{e}$ denote the Fermi levels for holes and electrons; (lower) 1D band diagrams of the STM tunnel junction with the STM tip placed at the positions indicated by the two green dashed lines. (e) Cross sections of SPV images in (b) across the interface of the $p-n$ junction. 
static condition. Figure 2(a) shows an SPV image of $V_{S}=$ $+2.5 \mathrm{~V}$, obtained over a $1000 \mathrm{~nm} \times 1000 \mathrm{~nm}$ area that includes $n^{+}-, n-$, and $p$-type layers, as indicated in Fig. 2(b). Since TIBB under positive $V_{S}$ is large (negligible) for the $n$-type ( $p$-type) region, the change in SPV across the $p$ - $n$ junction is clear. Furthermore, the difference in the SPV between $n^{+}$- and $n$-type layers due to the difference in the dopant concentration is also clear. A line profile of the blue line in Fig. 2(a) is plotted in Fig. 2(b), where the averaged values of SPV are $0.50 \mathrm{~V}$ for the $n^{+}$-GaAs region and $0.85 \mathrm{~V}$ for the $n$-GaAs region, respectively. To examine the validity of these values, we calculated the electrostatic potential beneath the STM tip in the three-dimensional geometry, assuming a parabolic tip [11,13]. The calculated values are 0.58 and $0.82 \mathrm{~V}$ for $n^{+}$-GaAs and $n$-GaAs, respectively, which are in excellent agreement with the values obtained experimentally.

Figures 2(c) and 2(d) are the SPV images obtained at the $p$ - $n$ junction interface with positive $[(\mathrm{c})+2.5 \mathrm{~V}]$ and negative [(d) $-2.5 \mathrm{~V}] V_{S}$, respectively. The SPV in the space charge region ( $p$ - $n$ junction interface) is almost zero as shown using the line profiles of these SPV images in Fig. 2(e). Zero SPV in the space charge region appears to be anomalous because TIBB should be induced under both polarities of the $V_{S}$ at the $p-n$ interface [14].

In general, the electric field between the tip and the semiconductor surface modifies the electrostatic potential in the semiconductor sample. Because of the modified potential field, photoexcited carriers drift and accumulate on the sample surface beneath the STM tip, resulting in nonequilibrium carrier distribution that reduces the electrostatic potential field. However, in the space charge region, photoexcited carriers flow across the area due to the built-in electric field formed by the $p$ - $n$ junction and do not accumulate under the STM tip, resulting in the observed zero SPV. The SPV spectra obtained at different sample bias voltages were averaged in each area and summarized in Fig. 2(f). The difference between SPV spectra of the three different areas is clear, and the SPV spectrum for the space charge region is zero for all sample bias voltages.

By taking advantage of the above phenomenon, we can identify the position of the space charge region [green bar in Fig. 2(e)]. The experimental value of the width is $50 \mathrm{~nm}$, which is in good agreement with the calculated value, $45 \mathrm{~nm}$ [15]. As has been seen, nanoscale characteristics of the local band structure of the semiconductor material can be quantitatively determined using LM-STS.

Now, we employed this method to examine the mechanism of the carrier transport properties, of which a prediction was made from the results of the macroscopic scale for this sample. The relationship between the $p$ - $n$ diode current $I_{F}$ and the forward-bias voltage $V_{F}$ determined from the macroscopic current-voltage characteristics is expressed as

$$
I_{F} \approx \exp \left(\frac{q V_{F}}{\eta k T}\right)
$$

( $q$ : electronic charge, $k$ : Boltzmann constant, $T$ : temperature, $\eta$ : ideality factor).

There are four different voltage regions, as indicated by the linear fits of the curve with different values of $\eta$, as shown in Fig. 3(c). The mechanism that explains each of the four regions was introduced as related to the characteristics of the minority-carrier diffusion properties [16] as follows: (1) $\eta=2\left(V_{F}: 0-0.4 \mathrm{~V}\right.$, blue line) Recombination region: minority carriers mostly recombine in the space charge area. (2) $\eta=1\left(V_{F}: 0.4-0.6 \mathrm{~V}\right.$, green line) Ideal diffusion region: minority carriers start to diffuse into the neutral area. (3) $\eta=2\left(V_{F}: 0.6-0.8 \mathrm{~V}\right.$, orange line) High injection region: injected-minority-carrier density becomes comparable to the majority-carrier density. (4) $\eta=2\left(V_{F}: 0.8 \mathrm{~V}-\right.$, red arrow $)$ Series resistance region: minority-carrier diffusion length apparently becomes longer than those in (2) and (3), because of the electric field by the $V_{F}$ leaked into the neutral $n$ - and $p$-type areas.

Although Eq. (1) is the important basis in the analysis of semiconductor devices, the mechanism was characterized from the empirically obtained results, that is, macroscopic current-voltage relationship as shown in Fig. 3(c).

Figures 3(b) are a series of SPV mappings obtained at the $p-n$ junction with different forward-bias voltages. Here, the additional bias voltage, $V_{F}$, was applied to the sample via the $n^{+}$- and $p$-side electrodes, as illustrated in Fig. 3(a). Since the $V_{S}$ is positive $(+2.5 \mathrm{~V})$, the SPV is large for the $n$-type area. When $V_{F}$ is zero, the SPV image is the same as that shown in Fig. 2(c), as expected. However, when the $V_{F}$ is applied, the SPV in the vicinity of the interface is reduced with increasing $V_{F}$. Since the decrease in SPV is related to the decrease in TIBB that is caused by the screening of the electrostatic field below the STM tip, the observed change is directly related to the change in the amount of the excess carriers below the STM tip. This is due to the injection of minority holes into the $n$-type neutral region, as illustrated in Fig. 3(d).

As seen in Fig. 3(b), the SPV image at $0.4 \mathrm{~V}$ is similar to that at $0 \mathrm{~V}$, because only a small number of minority carriers diffused into the neutral $n$-type area in the recombination region $(0.4 \mathrm{~V})$. In contrast, the SPV at the interface began to decrease in the ideal diffusion region $(0.5$ and $0.6 \mathrm{~V})$, and this decrease became prominent in the high injection region $(0.7 \mathrm{~V} \sim)$, as predicted. This is the first demonstration of the direct visual observation of carrier transport in a $p-n$ junction.

Let us look into the details of the minority-carrier diffusion. The variation of SPV with $V_{F}$ at a point $x$, depending on the excess minority-carrier density $\rho_{\mathrm{ex}}=\rho\left(x, V_{F}\right)$, is generally expressed in a simple logarithmic relationship of

$$
\Delta \mathrm{SPV}=C \ln \left(\rho_{\mathrm{ex}} / \rho_{0}\right),
$$

where $C$ is a constant needed for the conversion of units and $\rho_{0}$ is an arbitrary carrier density used for normalization [8]. The excess minority-carrier density as a function of the 
distance from the $p$ - $n$ junction interface is expressed as

$$
\rho_{\mathrm{ex}}=\rho\left(x_{0}, V_{F}\right) \exp \left[-\left(x-x_{0}\right) / L_{p}\right],
$$

where $x_{0}$ is the location of the boundary between the neutral region and the space charge region in the $n$-type layer and $L_{p}$ is the diffusion length of the minority hole in the direction perpendicular to the interface [17]. By substituting $\rho_{\text {ex }}$ in Eq. (3) into Eq. (2), we obtain the variation of SPV as a function of the distance and the minoritycarrier density as

$$
\Delta \mathrm{SPV}=C\left[-\frac{\left(x-x_{0}\right)}{L_{p}}+\ln \left(\frac{\rho\left(x_{0}, V_{F}\right)}{\rho_{0}}\right)\right]
$$

Experimental results can be analyzed using Eq. (4). Figure 3(e) shows the cross-sectional profiles of the SPV images in Fig. 3(b). The SPV varies almost linearly with the distance from the interface and shifts vertically with an increase in the minority-carrier injection rate as expected from Eq. (4). To obtain $L_{P}$, we estimated the value of $C$ using Eq. (2) [17] and analyzed the slopes of the crosssection profiles of SPV by linear fitting [color lines in Fig. 3(e)]. The values of $L_{P}$ obtained with different forward-bias voltages are listed in Table I. $L_{P}$ is insensitive to the $V_{F}$ up to the high injection region $\leq 0.7 \mathrm{~V}$ ), while an extension of $L_{P}$ is clear for the series resistance region $(0.8 \mathrm{~V} \leq)$. In the series resistance region, as the name indicates, the series resistances formed by the neutral $n$ and $p$-type regions become comparable to that of the space charge region due to the significant reduction of the built-in potential. Therefore, the voltage drops within the $n$-type region; hence, the electric field appearing in the region induces the drift of minority holes in its direction, resulting in the apparent extension of $L_{P}$ [16].

Since hole mobility at $300 \mathrm{~K}$ is phonon limited, the maximum diffusion length $L_{P}$ in the Si-doped $n$-GaAs sample with the same doping concentration [18] is $\sim 400 \mathrm{~nm}$, and decreases with the influence, for example, of the distribution of dopants and defects. The obtained values with the $V_{F}$ below $0.7 \mathrm{~V}$ ( up to the high injection region) are consistent with the prediction.

In conclusion, the doping characteristics and carrier transport in a GaAs $p$ - $n$ junction were visualized with a

TABLE I. Minority-hole diffusion length $L_{p}$ obtained from the SPV measurement [Fig. 3(e)].

\begin{tabular}{cc}
\hline \hline Forward-bias voltage $(\mathrm{V})$ & Hole diffusion length $(\mathrm{nm})$ \\
\hline 0.5 & $181 \pm 28$ \\
0.6 & $183 \pm 15$ \\
0.7 & $180 \pm 9$ \\
0.8 & $222 \pm 12$ \\
0.9 & $429 \pm 32$ \\
\hline
\end{tabular}

$\sim 10 \mathrm{~nm}$ spatial resolution, using LM-STS. The empirically obtained $I-V$ characteristic properties were successfully examined, for the first time, on the nanoscale. These results provide a solid basis for elucidating the mechanism of the carrier transport properties predicted by using the macroscopic analysis. Combining this visualization method with the atomic-scale structural analyses will offer further insight into the behavior of semiconductor devices under operating conditions.

This work was supported in part by a Grant-in-Aid for scientific research from the Ministry of Education, Culture, Sports, Science, and Technology of Japan. We thank Ms. Rie Yamashita, in our group at University of Tsukuba, for her help in preparing this Letter.

*Electronic address: http://dora.bk.tsukuba.ac.jp/

[1] W. D. Rau, P. Schwander, F. H. Baumann, W. Hoppner, and A. Ourmazd, Phys. Rev. Lett. 82, 2614 (1999).

[2] V. V. Zavyalov, J.S. McMurray, and C.C. Williams, J. Appl. Phys. 85, 7774 (1999).

[3] R. Shikler, T. Meoded, N. Fried, and Y. Rosenwaks, Appl. Phys. Lett. 74, 2972 (1999).

[4] N. Duhayon et al., J. Vac. Sci. Technol. B 22, 385 (2004).

[5] D. Ban, E. H. Sargent, S. Dixon-Warren, IEEE J. Quantum Electron. 40, 865 (2004).

[6] M. A. Topinka et al., Nature (London) 410, 183 (2001).

[7] O. Takeuchi, S. Yoshida, and H. Shigekawa, Appl. Phys. Lett. 84, 3645 (2004).

[8] L. Kronik and Y. Shapira, Surf. Sci. Rep. 37, 1 (1999).

[9] S. Grafström, J. Appl. Phys. 91, 1717 (2002).

[10] M. McEllistrem, G. Haase, D. Chen, R. J. Hamers, Phys. Rev. Lett. 70, 2471 (1993).

[11] R. M. Feenstra, J. Vac. Sci. Technol. B 21, 2080 (2003).

[12] Dopant concentration of each layer is confirmed by secondary ion mass spectrometer (SIMS).

[13] Calculations were performed using the SEMITIP program, developed by R. M. Feenstra [11]. Parameters used: work function of $W$ tip $=4.6 \mathrm{eV}$, applied bias voltage $=$ $+2.5 \mathrm{~V}$, radius of tip $=20 \mathrm{~nm}$, tip-sample distance $=$ $1 \mathrm{~nm}, T=300 \mathrm{~K}$, tip shank opening angle $\theta=90$.

[14] N. D. Jäger et al., Phys. Rev. B 67, 165307 (2003).

[15] The width of the space charge region $W$ was calculated using the equation [16] $W=\sqrt{\frac{2 \varepsilon_{s}}{q}\left[\frac{N_{A}+N_{D}}{N_{A} N_{D}}\right] V_{b i}}$, where $N_{A}$ and $N_{D}$ are acceptor and donor doping densities, respectively. $\varepsilon_{s}$ is the dielectric constant of GaAs, and $V_{b i}$ is the built-in potential of the $p-n$ junction.

[16] S.M. Sze, Physics of Semiconductor Devices (Wiley, New York, 1981), 2nd ed.

[17] $C$ was derived by subtraction of the two SPV values $(0.6 \mathrm{~V}$ and $0.7 \mathrm{~V})$ using Eq. (2): $\Delta \operatorname{SPV}\left(x_{0}, 0.7 \mathrm{~V}\right)-$ $\Delta \operatorname{SPV}\left(x_{0}, 0.6 \mathrm{~V}\right)=C \ln \left[\rho\left(x_{0}, 0.7 \mathrm{~V}\right) / \rho\left(x_{0}, 0.6 \mathrm{~V}\right)\right]$. Provided that $\rho\left(x, V_{F}\right)$ is proportional to the macroscopic $p$ - $n$ diode current with the forward-bias voltage $V_{F}, I_{V}$, and $\rho\left(x_{0}, 0.7 \mathrm{~V}\right) / \rho\left(x_{0}, 0.6 \mathrm{~V}\right)$ is equal to $I_{0.7 \mathrm{~V}} / I_{0.6 \mathrm{~V}}$, these values can be obtained from Fig. 3(c).

[18] B. Sieber, Mater. Sci. Eng. B 24, 35 (1994). 